Research Article

\title{
Linear Barycentric Rational Method for Solving Schrodinger Equation
}

\author{
Peichen Zhao $\mathbb{D}^{1}$ and Yongling Cheng $\mathbb{D}^{2}$ \\ ${ }^{1}$ School of Mathematics and Statistics, Heze University, Heze 274015, China \\ ${ }^{2}$ College of Science, North China University of Science and Technology, Tangshan 063210, China
}

Correspondence should be addressed to Yongling Cheng; chengyongling1129@163.com

Received 18 February 2021; Accepted 7 October 2021; Published 27 October 2021

Academic Editor: Ram Jiwari

Copyright @ 2021 Peichen Zhao and Yongling Cheng. This is an open access article distributed under the Creative Commons Attribution License, which permits unrestricted use, distribution, and reproduction in any medium, provided the original work is properly cited.

\begin{abstract}
A linear barycentric rational collocation method (LBRCM) for solving Schrodinger equation (SDE) is proposed. According to the barycentric interpolation method (BIM) of rational polynomial and Chebyshev polynomial, the matrix form of the collocation method (CM) that is easy to program is obtained. The convergence rate of the LBRCM for solving the Schrodinger equation is proved from the convergence rate of linear barycentric rational interpolation. Finally, a numerical example verifies the correctness of the theoretical analysis.
\end{abstract}

\section{Introduction}

Schrodinger equation (SDE) is widely used in atomic physics, nuclear physics and solid physics, quantum mechanics, and so on. SDE is only applicable to nonrelativistic particles with low velocity, and there is no description of particle spin. In this paper, we are concerned with solving the numerical solution of the SDE:

$$
\begin{aligned}
i h \frac{\partial \varphi(x, t)}{\partial t} & =-\frac{h^{2}}{2 m} \frac{\partial^{2} \varphi(x, t)}{\partial x^{2}}+V(x, t) \varphi(x, t)+f(x, t) \\
\varphi(x, 0) & =g_{1}(x), \varphi(x, t)=g_{2}(x), x \in(a, b), \\
\varphi(a, t) & =h_{1}(t), \varphi(b, t)=h_{2}(t), \quad 0<t<T
\end{aligned}
$$

where $h$ is reduced Planck constant and $m$ denotes quality. In [1], the fractional Schrodinger-Choquard equation with blow-up criteria and instability of normalized standing waves is studied. In [2], the finite-difference time-domain (FDTD) method is studied to solve SDE. In [3], nonlinear magnetic Schrodinger-Poisson type equation is studied. In [4], high-order multiscale discontinuous Galerkin method for one-dimensional stationary SDEs with oscillating solutions is presented. In [5], sixth-order nonlinear SDE is concerned by factorization formula and an analytical method. In [6], nonlinear SDEs are solved by the iterative method. In [7], the two-dimensional Klein-Gordon SDEs are solved by linear compact alternating direction implicit (CADI) scheme.

For getting the equidistant node of the barycentric formula, Floater [8-10] has proposed a reasonable interpolation method; in particular, equidistant distribution nodes and the quasi-equidistant nodes have high numerical stability and accuracy of interpolation [11, 12]. In [13, 14], the linear barycentric rational collocation method (LBRCM) have been used to solve the integro-differential equation. Wang et al. [15-17] have expanded the application fields of the collocation method (CM), such as initial value problems, plane elasticity problems, and nonlinear problems. LBRCM for solving heat conduction equation and biharmonic equation are studied in $[18,19]$.

In this paper, a LBRCM for solving SDE is proposed. According to the barycentric interpolation method (BIM) of rational polynomial and Chebyshev polynomial, the matrix form of the collocation method that is easy to program is obtained. The convergence rate of the LBRC method for 
solving the telegraph equation is proved from the convergence rate of linear barycentric rational interpolation (LBRI). Finally, a numerical example verifies the correctness of the theoretical analysis.

The remaining of this paper is planned as follows. Section 2 presents the differentiation matrices, CM for SDE, and the matrix form of CM. In Section 3, the convergence rate is proved. Finally, a numerical example verifies the theoretical analysis.

\section{Differentiation Matrices of SDE}

We partition the interval $[a, b]$ and $[0, T]$ into $a=x_{0}<x_{1}<\cdots<x_{m}=b$ and $0=t_{0}<t_{1}<\cdots<t_{n}=T$ with $h_{i}=x_{i}-x_{i-1}, \quad i=0,1, \ldots, m$, and $\tau_{j}=t_{j}-t_{j-1}, \quad j=0,1$, $\ldots, n$, for the uniform partition with $h=(b-a) / m$ and $\tau=T / n$. For $\Omega=[a, b] \times[0, T]$ with $\left(x_{i}, t_{j}\right), \quad i=0,1$, $\ldots, m$ and $j=0,1, \ldots, n$ will be the uniform partition.

Consider the barycentric interpolation function (BIF) as

$$
\varphi\left(x_{i}, t\right)=\varphi\left(x_{i}\right), \quad i=0,1, \ldots, m,
$$

and its barycentric interpolation approximation is

$$
\varphi(x, t)=\sum_{i=0}^{m} \sum_{j=0}^{n} r_{i}(x) r_{j}(t) \varphi_{i j},
$$

where

$$
\begin{aligned}
r_{i}(x) & =\frac{w_{i} /\left(x-x_{i}\right)}{\sum_{k=0}^{n} w_{k} /\left(x-x_{k}\right)}, \\
w_{i} & =\sum_{k_{1} \in J_{i}}(-1)^{i-d_{1}} \prod_{h_{1}=k_{1}, h_{1} \neq i}^{k_{1}+d_{1}} \frac{1}{\left|x_{i}-x_{h_{1}}\right|},
\end{aligned}
$$

where $J_{i}=\left\{k_{1} \in I_{m}: i-d_{1} \leq k_{1} \leq i\right\}, I_{m}=\left\{0, \ldots, m-d_{1}\right\}$, and

$$
r_{j}(t)=\frac{w_{j} /\left(t-t_{j}\right)}{\sum_{k=0}^{n} w_{k} /\left(t-t_{k}\right)}, w_{j}=\sum_{k_{2} \in J_{j}}(-1)^{j-d_{2}} \prod_{h_{2}=k_{2}, h_{2} \neq j}^{k_{2}+d_{2}} \frac{1}{\left|t_{j}-t_{h_{2}}\right|},
$$

where $J_{j}=\left\{k_{2} \in I_{n}: j-d_{2} \leq k_{2} \leq j\right\}, I_{n}=\left\{0, \cdots, n-d_{2}\right\}$ is the basis function, and $\varphi_{i j}$ is the value at point $\varphi\left(x_{i}, t_{j}\right)$. Combining equations (1) and (5), we obtain

$$
i\left(I_{m} \otimes D^{(1)}\right)\left[\begin{array}{c}
\varphi_{0} \\
\vdots \\
\varphi_{m}
\end{array}\right]+\left(C^{(2)} \otimes I_{n}\right)\left[\begin{array}{c}
\varphi_{0} \\
\vdots \\
\varphi_{m}
\end{array}\right]-I_{n}\left[\begin{array}{c}
\varphi_{0} \\
\vdots \\
\varphi_{m}
\end{array}\right]=\left[\begin{array}{c}
f_{0} \\
\vdots \\
f_{m}
\end{array}\right],
$$

and then, we have

$$
\left[i I_{m} \otimes D^{(1)}+\left(C^{(2)} \otimes I_{n}\right)-I_{m} \otimes I_{n}\right] \Phi=F,
$$

and

$$
L \Phi=F
$$

where

$$
L=i I_{m} \otimes D^{(1)}+\left(C^{(2)} \otimes I_{n}\right)-I_{m} \otimes I_{n}
$$

and

$$
D_{i j}^{(1)}= \begin{cases}\frac{w_{j} / w_{i}}{t_{i}-t_{j}}, & i \neq j, \\ -\sum_{k \neq i} D_{i k}^{(1)}, & i=j,\end{cases}
$$

and

$$
C_{i j}^{(1)}= \begin{cases}\frac{w_{j} / w_{i}}{x_{i}-x_{j}}, & i \neq j, \\ -\sum_{k \neq i} C_{i k}^{(1)}, & i=j,\end{cases}
$$

$$
C_{i j}^{(2)}= \begin{cases}2 C_{i j}^{(1)}\left(D_{i i}^{(1)}-\frac{1}{x_{i}-x_{j}}\right), & i \neq j, \\ -\sum_{k \neq i} C_{i k}^{(2)}, & i=j,\end{cases}
$$

and $\otimes$ is Kronecher product of matrix. In the following, we define the Kronecher product of matrix $A=\left(a_{i j}\right)_{m \times n}$ and $B=\left(b_{i j}\right)_{k \times l}$ as

$$
A \otimes B=\left(a_{i j} B\right)_{m \cdot k \times n \cdot l}
$$

where

$$
a_{i j} B=\left[\begin{array}{cccc}
a_{i j} b_{11} & a_{i j} b_{12} & \cdots & a_{i j} b_{1 l} \\
a_{i j} b_{21} & a_{i j} b_{22} & \cdots & a_{i j} b_{2 l} \\
\vdots & \vdots & \vdots & \vdots \\
a_{i j} b_{k 1} & a_{i j} b_{k 2} & \cdots & a_{i j} b_{k l}
\end{array}\right] .
$$

\section{Convergence Rate and Error Analysis}

The barycentric rational interpolants of function (BRIF) $\phi(x)$ with $r(x)$ and its error convergence rate is

$$
\begin{aligned}
e(x):= & \varphi(x)-r_{n}(x)=\left(x-x_{i}\right) \ldots \\
& \cdot\left(x-x_{i+d}\right) \varphi\left[x_{i}, x_{i+1}, \ldots x_{i+d}, x\right],
\end{aligned}
$$

and

$$
e(x)=\frac{\sum_{i=0}^{n-d} \lambda_{i}(x)\left(\varphi(x)-r_{n}(x)\right)}{\sum_{i=0}^{n-d} \lambda_{i}(x)}=\frac{A(x)}{B(x)}=O\left(h^{d+1}\right),
$$

where 


$$
A(x):=\sum_{i=0}^{n-d}(-1)^{i} \varphi\left[x_{i}, x_{i+1}, \ldots x_{i+d}, x\right]
$$

and

$$
B(x)=\sum_{i=0}^{n-d} \lambda_{i}(x)
$$

where

$$
\lambda_{i}(x)=\frac{(-1)^{i}}{\left(x-x_{i}\right) \cdots\left(x-x_{i+d}\right)} .
$$
[11].

The following Lemma was proved by Jean-Pau Berrut in
Lemma 1 (see [11]), For $e(x)$ defined in (16), we have

$$
\begin{cases}|e(x)| \leq C h^{d+1}, & \varphi \in C^{d+2}[a, b] \\ \left|e^{\prime}(x)\right| \leq C h^{d}, & \varphi \in C^{d+3}[a, b] \\ \left|e^{\prime \prime}(x)\right| \leq C h^{d-1}, & \varphi \in C^{d+4}[a, b], d \geq 1 .\end{cases}
$$

For the BRIF $\varphi(x, t)$ with $r(x, t)$, we can get the barycentric rational interpolation (BRI):

$$
r_{n}(x, t)=\frac{\sum_{i=0}^{m} \sum_{j=0}^{n} w_{i j} /\left[\left(x-x_{i}\right)\left(t-t_{j}\right)\right] \varphi_{i j}}{\sum_{i=0}^{m} \sum_{j=0}^{n} w_{i j} /\left(x-x_{i}\right)\left(t-t_{j}\right)},
$$

where

$$
w_{i j}=\sum_{k_{1} \in J_{i}} \sum_{k_{2} \in J_{j}}(-1)^{i-d_{1}+j-d_{2}} \prod_{h_{1}=k_{1}, h_{1} \neq i}^{k_{1}+d_{1}} \frac{1}{\left|x_{i}-x_{h_{1}}\right|} \prod_{h_{2}=k_{2}, h_{2} \neq j}^{k_{2}+d_{2}} \frac{1}{\left|t_{j}-t_{h_{2}}\right|},
$$

and $J_{i}=\left\{k_{1} \in I_{m}: i-d_{1} \leq k_{1} \leq i\right\}, I_{m}=\left\{0 \ldots, m-d_{1}\right\}$,

$$
J_{j}=\left\{k_{2} \in I_{n}: j-d_{2} \leq k_{2} \leq j\right\}, I_{n}=\left\{0 \ldots, n-d_{2}\right\} .
$$

By the error term of Newton-Cotes rule for two-dimensional function, we have

$$
\begin{aligned}
e(x, t):= & \varphi(x, t)-r(x, t)=\varphi(x, t)-r_{n}(x, t) \\
= & \left(x-x_{i}\right) \cdots\left(x-x_{i+d_{1}}\right) \varphi\left[x_{i}, x_{i+1}, \cdots x_{i+d_{1}}, x\right] \\
& +\left(t-t_{j}\right) \cdots\left(t-t_{j+d_{2}}\right) \varphi\left[t_{j}, t_{j+1}, \cdots t_{j+d_{2}}, t\right] .
\end{aligned}
$$

The following theorem has been proved in reference by Li in [18].

Theorem 1. For $e(x, t)$ defined in (25) and $\varphi(x, t) \in C^{d_{1}+2}[a, b] \times C^{d_{2}+2}[0, T]$, we have

$$
|e(x, t)| \leq C\left(h^{d_{1}+1}+\tau^{d_{2}+1}\right) .
$$

Corollary 1. For e $(x, t)$ defined in (25),

$$
\begin{cases}\left|e_{x}(x, t)\right| \leq C\left(h^{d_{1}}+\tau^{d_{2}+1}\right), & \varphi(x, t) \in C^{d_{1}+3}[a, b] \times C^{d_{2}+2}[0, T], \\ \left|e_{t}(x, t)\right| \leq C\left(h^{d_{1}+1}+\tau^{d_{2}}\right), & \varphi(x, t) \in C^{d_{1}+2}[a, b] \times C^{d_{2}+3}[0, T], \\ \left|e_{x x}(x, t)\right| \leq C\left(h^{d_{1}-1}+\tau^{d_{2}+1}\right), & \varphi(x, t) \in C^{d_{1}+4}[a, b] \times C^{d_{2}+2}[0, T], \quad d_{1} \geq 1 .\end{cases}
$$

This corollary can be obtained similarly as Theorem 1, where we omit it.

Let $\varphi(x, t)$ be the solution of (1) and $\varphi\left(x_{m}, t_{n}\right)$ be the numerical solution; then, we have

$$
D \varphi\left(x_{m}, t_{n}\right)=f(x, t),
$$

and

$$
\lim _{m, n \longrightarrow \infty} D \varphi\left(x_{m}, t_{n}\right)=f(x, t) .
$$

According to the above lemma, the following theorem can be proved.

Theorem 2. Let $\varphi\left(x_{m}, t_{n}\right): D \varphi\left(x_{m}, t_{n}\right)=f(x, t)$ and $f(x) \in C[a, b]$; we have

$$
\left|\varphi(x, t)-\varphi\left(x_{m}, t_{n}\right)\right| \leq C\left(h^{d_{1}-1}+\tau^{d_{2}}\right) .
$$

Proof. As 
TABLe 1: Convergence rate of equidistant nodes with different $d_{1}=7$ and $t=2$.

\begin{tabular}{|c|c|c|c|c|c|c|c|c|}
\hline$n$ & $d_{2}=2$ & & $d_{2}=3$ & & $d_{2}=4$ & & $d_{2}=5$ & \\
\hline $8 \times 8$ & $6.1237 e-01$ & & $8.7824 e-01$ & & $7.4433 e-01$ & & $1.1222 e+00$ & \\
\hline $16 \times 16$ & $1.7534 e-01$ & 1.8042 & $1.0471 e-01$ & 3.0682 & $3.0341 e-02$ & 4.6166 & $4.9307 e-02$ & 4.5084 \\
\hline $32 \times 32$ & $3.3624 e-02$ & 2.3826 & $6.6239 e-03$ & 3.9826 & $2.3415 e-03$ & 3.6957 & $7.0714 e-04$ & 6.1236 \\
\hline $64 \times 64$ & $6.0006 e-03$ & 2.4863 & $4.5724 e-04$ & 3.8567 & $1.1369 e-04$ & 4.3643 & $1.0298 e-05$ & 6.1016 \\
\hline
\end{tabular}

TABle 2: Convergence rate of equidistant nodes with different $d_{2}=7$ and $t=2$.

\begin{tabular}{|c|c|c|c|c|c|c|c|c|}
\hline$n$ & $d_{1}=2$ & & $d_{1}=3$ & & $d_{1}=4$ & & $d_{1}=5$ & \\
\hline $8 \times 8$ & 1.6771 & & 1.6716 & & 1.6795 & & 1.6782 & \\
\hline $16 \times 16$ & $1.5313 e-02$ & 6.7751 & $1.6007 e-02$ & 6.7064 & $1.6217 e-02$ & 6.6944 & $1.6091 e-02$ & 6.7045 \\
\hline $32 \times 32$ & $1.4665 e-03$ & 3.3843 & $7.1109 e-05$ & 7.8144 & $8.4703 e-05$ & 7.5809 & $7.2416 e-05$ & 7.7957 \\
\hline $64 \times 64$ & $1.8040 e-04$ & 3.0231 & $2.9783 e-06$ & 4.5775 & $6.9138 e-07$ & 6.9368 & $2.4334 e-07$ & 8.2172 \\
\hline
\end{tabular}

Table 3: Convergence rate of quasi-equidistant nodes with different $d_{1}=7$ and $t=2$.

\begin{tabular}{|c|c|c|c|c|c|c|c|c|}
\hline$n$ & $d_{2}=2$ & & $d_{2}=3$ & & $d_{2}=4$ & & $d_{2}=5$ & \\
\hline $8 \times 8$ & $5.1334 e-01$ & & $3.7631 e-01$ & & $1.1156 e-01$ & & $1.2259 e-01$ & \\
\hline $16 \times 16$ & $2.8133 e-02$ & 4.1896 & $1.1572 e-02$ & 5.0232 & $4.8063 e-03$ & 4.5368 & $2.5873 e-03$ & 5.5663 \\
\hline $32 \times 32$ & $2.8913 e-03$ & 3.2825 & $5.4800 e-04$ & 4.4003 & $1.4573 e-04$ & 5.0435 & $2.1084 e-05$ & 6.9392 \\
\hline $64 \times 64$ & $3.2935 e-04$ & 3.1340 & $3.0820 e-05$ & 4.1523 & $4.1594 e-06$ & 5.1308 & $2.5797 e-07$ & 6.3528 \\
\hline
\end{tabular}

TABle 4: Convergence rate of quasi-equidistant nodes with different $d_{2}=7$ and $t=2$.

\begin{tabular}{|c|c|c|c|c|c|c|c|c|}
\hline$n$ & $d_{1}=2$ & & $d_{1}=3$ & & $d_{1}=4$ & & $d_{1}=5$ & \\
\hline $8 \times 8$ & $2.8707 e-01$ & & $2.8566 e-01$ & & $2.8297 e-01$ & & $2.8323 e-01$ & \\
\hline $16 \times 16$ & $1.0263 e-02$ & 4.8059 & $5.7980 e-04$ & 8.9445 & $5.0888 e-04$ & 9.1191 & $5.0644 e-04$ & 9.1274 \\
\hline $32 \times 32$ & $6.1791 e-04$ & 4.0539 & $2.9283 e-06$ & 7.6293 & $1.1254 e-06$ & 8.8207 & $1.0428 e-06$ & 8.9237 \\
\hline $64 \times 64$ & $7.9511 e-05$ & 2.9582 & $1.3835 e-06$ & 1.0817 & $1.4398 e-06$ & - & $4.3847 e-06$ & - \\
\hline
\end{tabular}

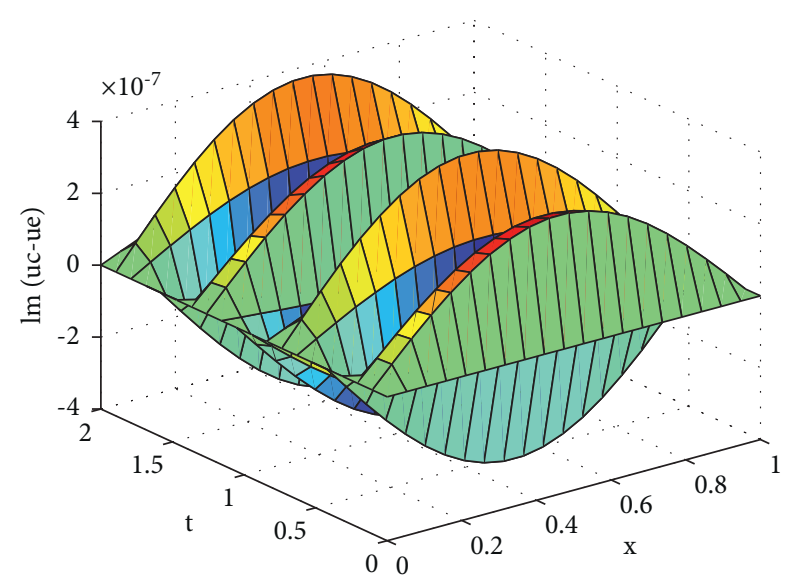

FIgURE 1: Error estimate of equidistant nodes with $t=2, m=n=19$, and $d_{1}=d_{2}=8$.

$$
\begin{aligned}
& D \varphi(x, t)-D \varphi\left(x_{m}, t_{n}\right) \\
= & i \varphi_{t}(x, t)-\varphi(x, t)+i \varphi_{x x}(x, t)-\left[i \varphi_{t}\left(x_{m}, t_{n}\right)-\varphi\left(x_{m}, t_{n}\right)+i \varphi_{x x}\left(x_{m}, t_{n}\right)\right] \\
= & i\left[\varphi_{t}(x, t)-\varphi_{t}\left(x_{m}, t_{n}\right)\right]+\left[-\varphi(x, t)+\varphi\left(x_{m}, t_{n}\right)\right]+i\left[\varphi_{x x}(x, t)-\varphi_{x x}\left(x_{m}, t_{n}\right)\right] \\
:= & i R_{1}(x, t)+R_{2}(x, t)+i R_{3}(x, t),
\end{aligned}
$$




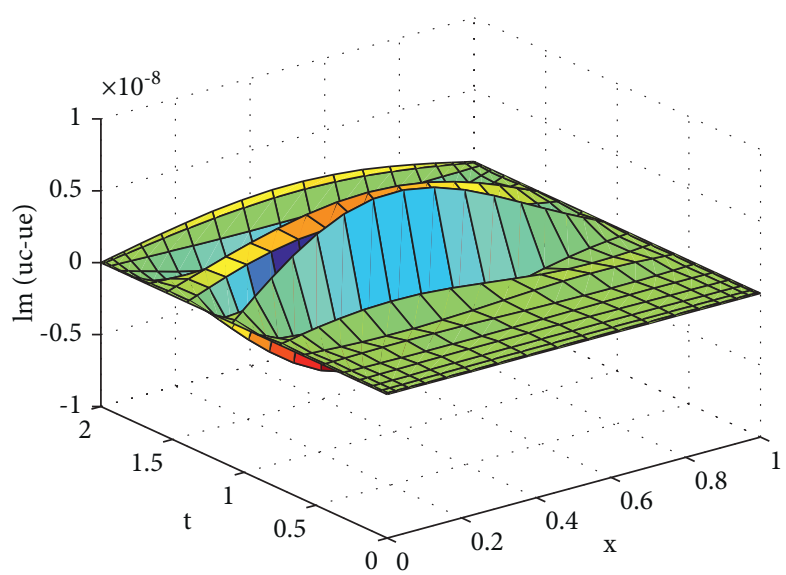

FIgURe 2: Error estimate of quasi-equidistant nodes with $t=2, m=n=19$, and $d_{1}=d_{2}=8$.

TABLE 5: Convergence rate of equidistant nodes with different $d_{1}=7$ and $t=1$.

\begin{tabular}{|c|c|c|c|c|c|c|c|c|}
\hline$n$ & $d_{2}=2$ & & $d_{2}=3$ & & $d_{2}=4$ & & $d_{2}=5$ & \\
\hline $8 \times 8$ & $1.7995 e-03$ & & $4.6557 e-04$ & & $1.5050 e-04$ & & $1.4417 e-04$ & \\
\hline $16 \times 16$ & $5.5795 e-04$ & 1.6894 & $6.5769 e-05$ & 2.8235 & $1.1394 e-05$ & 3.7235 & $1.5421 e-06$ & 6.5467 \\
\hline $32 \times 32$ & $1.1570 e-04$ & 2.2698 & $5.2988 e-06$ & 3.6337 & $4.9129 e-07$ & 4.5355 & $2.8309 e-08$ & 5.7675 \\
\hline $64 \times 64$ & $2.1482 e-05$ & 2.4291 & $4.7396 e-07$ & 3.4828 & $2.3083 e-08$ & 4.4117 & $5.7039 e-10$ & 5.6332 \\
\hline
\end{tabular}

TABle 6: Convergence rate of equidistant nodes with different $d_{2}=7$ and $t=1$.

\begin{tabular}{|c|c|c|c|c|c|c|c|c|}
\hline$n$ & $d_{1}=2$ & & $d_{1}=3$ & & $d_{1}=4$ & & $d_{1}=5$ & \\
\hline $8 \times 8$ & $1.6168 e-03$ & & $5.1505 e-04$ & & $1.7042 e-04$ & & $9.0483 e-05$ & \\
\hline $16 \times 16$ & $1.9952 e-04$ & 3.0185 & $2.1403 e-05$ & 4.5888 & $8.2906 e-06$ & 4.3615 & $1.2726 e-06$ & 6.1518 \\
\hline $32 \times 32$ & $2.5369 e-05$ & 2.9754 & $1.2042 e-06$ & 4.1516 & $2.4768 e-07$ & 5.0649 & $1.4595 e-08$ & 6.4461 \\
\hline $64 \times 64$ & $3.4030 e-06$ & 2.8982 & $7.6096 e-08$ & 3.9842 & $8.0774 e-09$ & 4.9384 & $3.4093 e-10$ & 5.4198 \\
\hline
\end{tabular}

TABle 7: Convergence rate of quasi-equidistant nodes with different $d_{1}=7$ and $t=1$.

\begin{tabular}{|c|c|c|c|c|c|c|c|c|}
\hline$n$ & $d_{2}=2$ & & $d_{2}=3$ & & $d_{2}=4$ & & $d_{2}=5$ & \\
\hline $8 \times 8$ & $2.1572 e-03$ & & $2.1324 e-04$ & & $2.9588 e-05$ & & $1.1861 e-05$ & \\
\hline $16 \times 16$ & $1.7804 e-04$ & 3.5989 & $6.5585 e-06$ & 5.0230 & $9.5692 e-07$ & 4.9505 & $7.8014 e-08$ & 7.2482 \\
\hline $32 \times 32$ & $1.2864 e-05$ & 3.7907 & $4.1551 e-07$ & 3.9804 & $3.2481 e-08$ & 4.8807 & $1.0198 e-09$ & 6.2574 \\
\hline $64 \times 64$ & $1.3676 e-06$ & 3.2336 & $2.2121 e-08$ & 4.2314 & $8.0913 e-09$ & 2.0051 & $2.4516 e-08$ & - \\
\hline
\end{tabular}

TABle 8: Convergence rate of quasi-equidistant nodes with different $d_{2}=7$ and $t=1$.

\begin{tabular}{|c|c|c|c|c|c|c|c|c|}
\hline$n$ & $d_{1}=2$ & & $d_{1}=3$ & & $d_{1}=4$ & & $d_{1}=5$ & \\
\hline $8 \times 8$ & $2.1937 e-03$ & & $5.6367 e-04$ & & $7.8737 e-05$ & & $6.3534 e-06$ & \\
\hline $16 \times 16$ & $1.6769 e-04$ & 3.7094 & $3.6712 e-06$ & 7.2625 & $5.9101 e-07$ & 7.0577 & $8.7629 e-08$ & 6.1800 \\
\hline $32 \times 32$ & $1.4478 e-05$ & 3.5339 & $1.2449 e-07$ & 4.8821 & $1.2882 e--08$ & 5.5198 & $4.5441 e-10$ & 7.5913 \\
\hline $64 \times 64$ & $7.0525 e-06$ & 1.0376 & $6.1937 e-07$ & - & $2.2631 e-07$ & - & $1.0710 e-06$ & - \\
\hline
\end{tabular}


we have

$$
\begin{aligned}
& R_{1}(x, t)=\varphi_{t}(x, t)-\varphi_{t}\left(x_{m}, t_{n}\right), \\
& R_{2}(x, t)=\varphi(x, t)-\varphi\left(x_{m}, t_{n}\right), \\
& R_{3}(x, t)=\varphi_{x x}(x, t)-\varphi_{x x}\left(x_{m}, t_{n}\right) .
\end{aligned}
$$

As, for $R_{3}(x, t)$, we have

$$
\begin{aligned}
R_{3}(x, t)= & \varphi_{x x}(x, t)-\varphi_{x x}\left(x_{m}, t_{n}\right) \\
= & \varphi_{x x}(x, t)-\varphi_{x x}\left(x_{m}, t\right)+\varphi_{x x}\left(x_{m}, t\right)-\varphi_{x x}\left(x_{m}, t_{n}\right) \\
= & \frac{\sum_{i=0}^{m-d_{1}}(-1)^{i} \varphi_{x x}\left[x_{i}, x_{i+1}, \ldots, x_{i+d_{1}}, x, t\right]}{\sum_{i=0}^{m-d_{1}} \lambda_{i}(x)} \\
& +\frac{\sum_{j=0}^{n-d_{2}}(-1)^{j} \varphi_{x x}\left[t_{j}, t_{j+1}, \ldots, t_{j+d_{2}}, x_{m}, t\right]}{\sum_{j=0}^{n-d_{2}} \lambda_{j}(t)} \\
= & e_{x x}\left(x, t_{n}\right)+e_{x x}\left(x_{m}, t_{n}\right) .
\end{aligned}
$$

By the corollary, we obtain

$$
\left|R_{3}(x, t)\right| \leq\left|e_{x x}\left(x, t_{n}\right)+e_{x x}\left(x_{m}, t_{n}\right)\right| \leq C\left(h^{d_{1}+1}+\tau^{d_{2}+1}\right) .
$$

Similarly, for $R_{2}(x, t)$ and $R_{1}(x, t)$, we have

$$
R_{1}(x, t)=\varphi_{t}(x, t)-\varphi_{t}\left(x_{m}, t_{n}\right)=e_{t}\left(x, t_{n}\right)-e_{t}\left(x_{m}, t_{n}\right),
$$

$$
\left|R_{1}(x, t)\right| \leq\left|e_{t}\left(x, t_{n}\right)+e_{t}\left(x_{m}, t_{n}\right)\right| \leq C\left(h^{d_{1}+1}+\tau^{d_{2}}\right),
$$

and

$$
R_{2}(x, t)=\beta\left|\varphi(x, t)-\varphi\left(x_{m}, t_{n}\right)\right| \leq C\left(h^{d_{1}+1}+\tau^{d_{2}+1}\right) .
$$

Combining the identity equations (31), (34), (36), and (37), the conclusion of theorem is obtained.

\section{Numerical Examples}

Example 1. The SDE $a=0, b=1, t=2$, and

$$
f(x, t)=0,
$$

under condition $g_{1}(x)=\sqrt{2} \sin (\pi x)$; the analysis solution is

$$
\varphi(x, t)=\sqrt{2} e^{-i \pi^{2} t / 2} \sin (\pi x) .
$$

Tables 1 and 2 show the errors of the LBRCM for equidistant nodes of space variables and time variables.

Tables 3 and 4 show the errors of the LBRCM for quasiequidistant nodes of space variables and time variables.

Example 2. The SDE $a=0, b=1, t=1$, and

$$
f(x, t)=\left(x^{2}-2\right) e^{i \pi^{2} t / 4} \cos \left(\frac{\pi x}{2}\right)+2 \pi \sin \left(\frac{\pi x}{2}\right),
$$

under condition $g_{1}(x)=x^{2} \cos (\pi x / 2)$; the analysis solution is

$$
\varphi(x, t)=e^{-t} \sin x
$$

In Figures 1 and 2, the error estimate of equidistant and quasi-equidistant nodes with $t=2, m=n=19$, and $d_{1}$ $=d_{2}=8$ is presented. It can be seen from Figure 2 that the barycentric rational interpolation collocation method has higher accuracy in both quasi-equidistant and equidistant nodes conditions.

Tables 5 and 6 show the errors of the LBRCM for equidistant nodes of space variables and time variables.

Tables 7 and 8 show the errors of the LBRCM for quasiequidistant nodes of space variables and time variables.

\section{Conclusion}

In this paper, the LBRCM have been constructed to solve SDE, while the time variable and space variable are obtained at the same time. Numerical solution confirms the theorem analysis.

\section{Data Availability}

The data that support the findings of this study are available from the corresponding author upon reasonable request.

\section{Conflicts of Interest}

The authors declare that they have no conflicts of interest.

\section{Authors' Contributions}

This manuscript was written by Peicheng Zhao and Yongling Cheng. Some checks of grammar were given by Yongling Cheng.

\section{Acknowledgments}

The work of Yongling Cheng was supported by Natural Science Foundation of Hebei Province (Grant no. A2019209533).

\section{References}

[1] B. H. Feng, R. P. Chen, and J. Y. Liu, "Blow-up criteria and instability of normalized standing waves for the fractional Schrdinger-Choquard equation," Advances in Nonlinear Analysis, vol. 10, no. 1, pp. 331-330, 2021.

[2] P. Declee, A. V. Londersele, and H. Rogier, "Nonuniform and higher-order FDTD methods for the schrdinger equation," Journal of Computational and Applied Mathematics, vol. 381, no. 1, Article ID 113023, 2021.

[3] Y. L. Liu, X. Li, and J. Chao, "Multiplicity of concentrating solutions for a class of magnetic Schrdinger-Poisson type equation," Advances in Nonlinear Analysis, vol. 10, no. 1, pp. 131-151, 2021.

[4] B. Dong and W. Wang, "High-order multiscale discontinuous Galerkin methods for the one-dimensional stationary Schrdinger equation," Journal of Computational and Applied Mathematics, vol. 380, no. 15, Article ID 112962, 2020. 
[5] C. C. Liu, H. M. Wang, and Z. S. Feng, "Global solution for a sixth-order nonlinear Schrodinger equation," Journal of Mathematical Analysis and Applications, vol. 490, no. 2, Article ID 124327, 2020.

[6] C.-S. Liu, "A positivity preserving iterative method for finding the ground states of saturable nonlinear schrödinger equations," Journal of Scientific Computing, vol. 84, no. 3, 2020.

[7] J. Chen and F. Chen, "Maximum norm error estimates of a linear CADI method for the Klein-Gordon-Schrödinger equations," Computers and Mathematics with Applications, vol. 80, no. 5, pp. 1327-1342, 2020.

[8] M. S. Floater and K. Hormann, "Barycentric rational interpolation with no poles and high rates of approximation," Numerische Mathematik, vol. 107, no. 2, pp. 315-331, 2007.

[9] G. Klein and J.-P. Berrut, "Linear rational finite differences from derivatives of barycentric rational interpolants," SIAM Journal on Numerical Analysis, vol. 50, no. 2, pp. 643-656, 2012.

[10] G. Klein and J.-P. Berrut, "Linear rational finite differences from derivatives of barycentric rational interpolants," BIT Numerical Mathematics, vol. 52, no. 2, pp. 407-424, 2012.

[11] J.-P. Berrut, M. S. Floater, and G. Klein, "Convergence rates of derivatives of a family of barycentric rational interpolants," Applied Numerical Mathematics, vol. 61, no. 9, pp. 989-1000, 2011.

[12] J.-P. Berrut, S. A. Hosseini, and G. Klein, "The linear barycentric rational quadrature method for Volterra integral equations," SIAM Journal on Scientific Computing, vol. 36, no. 1, pp. A105-A123, 2014.

[13] J. Li and Y. L. Cheng, "Linear barycentric rational collocation method for solving second-order Volterra integro-differential equation," Computational and Applied Mathematics, vol. 39, no. 2, pp. 1-9, 2020.

[14] J. Li and Y. L. Cheng, "Numerical solution of Volterra integrodifferential equations with linear barycentric rational method," International Journal of Algorithms, Computing and Mathematics, vol. 6, 2020

[15] Z. Q. Wang and S. P. Li, Barycentric Interpolation Collocation Method for Nonlinear Problems, National Defense Industry Press, Beijing, China, 2015.

[16] Z. Q. Wang, Z. K. Xu, and J. Li, "Mixed barycentric interpolation collocation method of displacement-pressure for incompressible plane elastic problems," Chinese Journal of Applied Mechanics, vol. 35, no. 3, pp. 631-637, 2018.

[17] Z. Q. Wang, L. Zhang, and Z. K. Xu, "Barycentric interpolation collocation method based on mixed displacement-stress formulation for solving plane elastic problems," Chinese Journal of Applied Mechanics, vol. 35, no. 2, pp. 304-309, 2018.

[18] J. Li and Y. L. Cheng, "Linear barycentric rational collocation method for solving heat conduction equation," Numerical Methods for Partial Differential Equations, vol. 37, Article ID 22539, 2020.

[19] J. Li and Y. L. Cheng, "Barycentric rational method for solving biharmonic equation by depression of order," Numerical Methods for Partial Differential Equations, vol. 1C15, Article ID 22638, 2020. 